\title{
CP8-8 \\ FK228, histone deacetylase inhibitor - A novel drug for the treatment of prostate and re- nal cancers-
}

Department of Urology, Medicinal Biology Research Lab. ,Fujisawa Pharmaceutical Co. ,Ltd. Naoe Yoshinori, Inoue Takeshi, Sasakawa Yuka, Matsuo Masahiko, Mutoh Seitaro

FK228 is a cyclic peptide that was discovered in a screening program for agents that reverse the malignant phenotype of H-ras-transformed NIH3T3 cells. Recent studies indicate that FK228 is a potent histone deacetylase (HDAC) inhibitor. Acetylation and deacetylation of histones are catalyzed by specific enzyme families, histone acetyltransferases and HDACs, respectively. The balance of histone acetylation plays an important regulatory role in the transcriptional of many genes. Hyperacetylation of histone is associated with a condensed chromatin structure resulting in the repression of gene transcription, whereas acetylated histones are associated with a more open chromatin structure and activation of transcription.

The antitumor effects of FK 228 against human prostate cancer xenografts (PC-3 and DU-145) and renal cancer xenografts (RXF-631L and ACHN) implanted s. c. in nude mice were evaluated. FK228 exhibited marked antitumor activity against PC-3 and RXF-631L and moderate regression of DU-145. On the other hand, FK228 did not show antitumor activity against ACHN. The antiproliferative activity of FK228 on PC-3 was accompanied by induction of apoptosis and cell cycle arrest at $\mathrm{G} 2 / \mathrm{M}$ phase. As HDAC inhibitor induces hyperacetylation of histone and modulates gene expression, we examined the effect of FK228 on gene expression. FK228 induced the expression of p21 mRNA and repressed VEFG and bFGF mRNA, suggesting that these change in gene expression contribute to its strong antitumor effects.

In phase I trial, FK228 has shown efficacy in patients with both renal cell carcinoma and T cell lymphoma. The promising clinical antitumor activity of FK228 suggests that it may be potentially useful treatment for both hematological and solid tumors.

\section{CP8-9}

\section{NF- $\mathrm{KB}$ function inhibitor, DHMEQ as a treatment option for advanced urological cancer}

Department of Urology, Keio University, School of Medicine ${ }^{1)}$, Department ofApplied Chemistry, Faculty of Science and Technology, Keio University ${ }^{2)}$

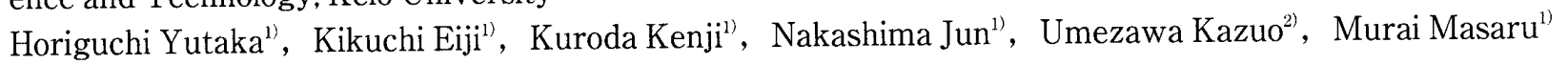

$\mathrm{NF}-\mathrm{kB}$ is a transcription factor that induces not only various cytokines such as IL-1, IL-2, IL-6, IL-8 and interferon- $\gamma$ but also anti-apoptotic proteins. It is well known that NF-KB is often constitutively activated in hormone-refractory prostate cancer as well as in advanced bladder cancer, which may increase expression of anti-apoptotic proteins, thereby decreasing the effectiveness of anti-tumor therapy and contributing to the development of chemo-resistant phenotypes. Recently, we have synthesized a novel NF-kB function inhibitor, a dehydroxymethyl derivative of epoxyquinomicin designated as DHMEQ. In our recent studies, we have investigated the effectiveness of DHMEQ as a treatment option for advanced urological cancers. Hormone-refractory prostate cancer cells have been known to produce multiple cytokines including IL-1 $\alpha$, IL-6 and granulocyte colony-stimulating factor, which are thought to play a crucial role in tumor growth and progression. It is suggested that TNF- $\alpha$ did not induce the cytotoxic effects on hormone-refractory prostate cancer cells, because NF-kB protective pathway prevents the cells to undergo apoptosis. These evidences support the hypothesis that NF-kB plays an important role in maintain chemoresistant nature of hormone-refractory prostate cancer. Therefore, approaches that inhibit NF- $\mathrm{kB}$ function may be beneficial in the treatment of chemo-resistant prostate cancer. The activity of NF-kB, evaluated by transient transfection of a reporter DNA containing a specific binding sequence for NF-KB, was inhibited by DHMEQ in human hormone-refractory prostate cancer cell lines. Statistically significant growth inhibition was achieved by $20 \mu \mathrm{g} / \mathrm{ml}$ of DHMEQ and marked levels of apoptosis were induced after DHMEQ administration in vitro. Electrophoretic mobility shift assay showed that DHMEQ completely inhibited NF-KB DNA binding activity. Furthermore, intraperitoneal administration of DHMEQ significantly inhibited pre-established subcutaneous xenograft prostate tumor growth in nude mice without any side effects. These results indicate the possibility of using DHMEQ, as a new treatment strategy against advanced urological cancer. 